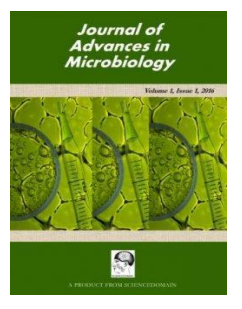

\title{
Occurrence of Bacterial Contaminants in Fin-fishes (Clarias gariepinus and Coptodon guineensis) from Humic Freshwater Ecosystem of Eniong River, Itu, Akwa Ibom State, Nigeria
}

\author{
Senyene I. Umana ${ }^{1 *}$, Ufokette C. Okon ${ }^{2}$, Mfoniso P. Uko ${ }^{1}$ and Maria P. Bassey ${ }^{1}$ \\ ${ }^{1}$ Department of Biological Sciences, Akwa Ibom State University, Nigeria. \\ ${ }^{2}$ Department of Veterinary, Ministry of Agriculture, Akwa Ibom State, Nigeria.
}

Authors' contributions

This work was carried out in collaboration between all authors. All authors read and approved the final manuscript.

Article Information

DOI: $10.9734 / J A M B / 2017 / 36529$ Editor(s):

(1) Charu Gupta, AlHRS, Amity University, UP, India. Reviewers:

(1) Francesca Andreoni, University of Urbino, Italy. (2) Wagner Loyola, Brazilian Agricultural Research Corporation, Brazil. (3) S. Thenmozhi, Periyar University, India. Complete Peer review History: http://www.sciencedomain.org/review-history/21373

Original Research Article

Received $30^{\text {th }}$ August 2017

Accepted $9^{\text {th }}$ October 2017

Published $13^{\text {th }}$ October 2017

\begin{abstract}
The occurrence of bacterial isolates in fin-fishes (Clarias gariepinus and Coptodon guineensis) from the humic ecosystem of Eniong River, Akwa Ibom State was investigated. The results obtained from pour plate analysis showed that the fin-fishes contained bacterial contaminants. The bacterial loads varied with the type of fin-fish and were much higher in fish intestines, when compared with the skin and gills. The heterotrophic bacterial loads obtained exceeded the $1.2 \times 10^{5} \mathrm{cfu} / \mathrm{g}$ limit recommended for fresh fishes. High and unsafe fecal coliform $\left(1.1 \pm 0.57 \times 10^{3} \mathrm{cfu} / \mathrm{g}-2 . \pm 0.26 \times 10^{3}\right.$ $\mathrm{cfu} / \mathrm{g})$ loads were also obtained. The culture-able bacteria species associated with the fin-fish samples include Staphylococcus sp, Klebsiella sp, Bacillus sp, Enterobacter, Streptococcus sp, Micrococcus sp, Lactobacillus, Serratia sp, Proteus sp, Salmonella sp, Shigella sp, and Escherichia coli. The percentage of occurrence of the isolates in the various fish samples was also found to vary with the fish species. Staphylococcus sp had the highest rate of occurrence of $63 \%$ and $44.4 \%$ in $C$. gariepinus and C. guineensis respectively while the least prevalent organism was Micrococcus sp
\end{abstract}


with $7.41 \%$, and Serratia and Shigella sp with $11.1 \%$ for C. gariepinus and C. guineensis respectively. Twelve bacterial species with variable virulent potentials were isolated. The analysis revealed that each of the isolates exhibited varying degree of virulence. Of the 12 bacterial isolates obtained from the various fish samples, ten (Bacillus cereus, Micrococcus sp, Streptococcus sp., Proteus sp, Serratia sp, Salmonella sp., Shigella sp., E. coli, Enterobacter sp and Lactobacillus sp had the potentials to produce lipases enzyme. This enzyme is responsible for breaking down lipids. Among the isolates Stahylococcus aureus was the most virulent. The results indicate poor microbiological quality. These call for proper processing of aquatic foods as well as routine monitoring.

Keywords: Clarias gariepinus; Coptodon guineensis; Contaminants; Freshwater and Humic.

\section{INTRODUCTION}

The aquatic ecosystem is made up of fresh water and marine water habitat. It is widely recognized that freshwater biodiversity and habitats are under serious threat [1-2] and that the level of threat exceeds or will soon exceed that in either terrestrial or marine ecosystems [3]. Dudgeon et al. [2] grouped the main threats under five interacting categories: over-exploitation; water pollution; flow modification; destruction or degradation of habitat; and invasion by exotic species. Environmental changes occurring at the global scale, such as nitrogen deposition, global warming and shifts in precipitation and runoff patterns, are superimposed upon all of these threat categories. The primary indirect-drivers of degradation and loss of habitat have been population growth and increasing economic development and the primary direct-drivers of degradation and loss include infrastructure development, land conversion, water withdrawal, pollution, over-harvesting and over-exploitation, and the introduction of invasive alien species [3]. In Africa the most immediate impacts are likely to include habitat degradation and flow modification due to the actions of development projects aimed at meeting the growing requirement for access to safe drinking water, improved sanitation, irrigation for agriculture, and hydropower.

Fish are susceptible to a wide variety of bacterial pathogens, most of which are capable of causing disease and are considered by some to be saprophytic in nature [4]. The microbiological diversity of fresh fish muscle depends on the fishing grounds and environmental factors around it [5]. It has been suggested that the type of micro-organisms that are found associated with particular fish depends on its habitat [6]. The bacterial pathogens associated with fish have been classified as indigenous and nonindigenous [7]. The non-indigenous contaminate the fish or the habitat one way or the other and examples include Escherichia coli, Clostridium botulinum, Shigella dysenteriae, Staphylococcus aureus, Listeria monocytogens and Salmonella. The indigenous bacterial pathogens are found naturally living in the fish habitat for example Vibrio sp and Aeromonas sp [8]. The bacteria from fish only become pathogenic when fishes are physiologically unbalanced, nutritionally deficient, or there are other stressors, i.e., poor water quality, overstocking, which allow opportunistic bacterial infections to prevail [9]. Pathogenic and potentially pathogenic bacteria associated with fish and shellfish include Mycobacterium, Streptococcus spp., Vibrio spp., Aeromonas spp., Salmonella spp. and others [4].

Integrated fish farming combines livestock production with fish farming. Animal manure is shed directly into a fish pond as fertilizer and supports the growth of photosynthetic organisms. While supplemental feeding affects fish growth directly, fertilization contributes to growth via the planktonic natural food. In addition to acting as food for fish, plankton perform other important functions in pond aquaculture: a net producer of dissolved oxygen, which is indispensable for fish growth and the most important sink of ammonianitrogen, which is excreted by fish [10]. The use of different kinds of livestock manure in fish production may increase the level of pathogenic bacteria causing a public health risk to the rural community [11]. It has been highlighted that fish consumption can be an important avenue for human pathogenic bacteria and other food borne diseases exposure to man [12]. Pathogens from fish can be transmitted to humans through both active and passive contact and may cause food borne diseases such as dysentery, typhoid fever, salmonellosis and cholera. The practice of livestock-fish farming needs to be placed in perspective with the likely health risks [13]. One of the risks involved in livestock integrated fish farming is possible transfer of pathogens between livestock and humans. Previous 
research has shown that, different kinds of livestock manure are contaminated with pathogenic bacteria such as Salmonella, Shigella, Pseudomonas, Vibrio, Streptococcus, and E. coli [14].

The transmission of these pathogens to people can be through improperly cooked food or the handling of the fish. There have been great economic losses reported due to food borne illness such as dysentery and diarrhea resulting from consumption of contaminated fish and such can be a problem to the immune compromised, children and elderly people. The microbial association with fish compromises safety and the quality for human consumption; particularly critical is when the micro-organisms are opportunistic and or pathogenic in nature [15].

All living organisms evolve. As multicellular organisms evolved on earth, bacteria, viruses and other microorganisms also adapted and evolved to populate the new niches provided by these larger organisms. While some microorganisms evolved a mutual but benign lifestyle in their multicellular hosts, others evolved a more aggressive and pathogenic lifestyle that ultimately harms or even kills the host. The fate of these microorganisms which are classified as microbial pathogens is dictated by their virulence and virulence factors (i.e. ability to survive, multiply and cause infection within the host).

Virulence factors are molecules expressed and or secreted by pathogens (bacteria, viruses, fungi and protozoa) that enable them colonize a niche in the host (this includes attachment to cells), evade and or inhibit host immune response, enter and exit from cells (applied to intercellular pathogens) and obtain nutrition from the host cell. These evolutions are triggered by intrinsic or extrinsic factors and may aid the organism to acquire more or develop their virulence factors. For instance, mutations which may occur due to mistakes in DNA replication or mutagenic conditions in the environment may be beneficial to the organism and those mutations that benefit the organism are maintained and passed on to succeeding generations of progeny. Although most bacterial virulence factors are chromosomally encoded and intrinsic to the bacteria (e.g. capsule and endotoxins), bacteria do not depend solely on the occasional random mutation to improve their fitness but have evolved mechanisms for acquiring genes directly from other bacteria through contact-mediated transfer of DNA (conjugation) and indirectly via bacteriophage vectors (transduction) or the uptake of naked DNA from their surroundings (transformation) [16].

Studies on the occurrence of bacterial contaminants in fish produce from some freshwater ecosystem have been conducted by some researchers [15,17-18] but little or no work has been done on humic fresh water ecosystem, hence, the need for the present study to provide information on ocurrence of bacterial contaminants of fin-fishes (Clarias gariepinus and Coptodon guineensis) from humic freshwater ecosystem of Eniong River, Itu, Akwa Ibom State.

\section{MATERIALS AND METHODS}

\subsection{Study Area}

The study area is a humic ecosystem of Eniong River, a tributary of the middle course of the Cross River located in South-Eastern coast of the Niger Delta region of Nigeria. The ecosystem is home to diverse species of fish resources and supports remarkable populations of fin-fishes including Clarias buthupogon and Coptodon guineensis (Plates I - II) that are widely consumed by the catchment communities in Itu Local Government Area of Akwa Ibom State.

\subsection{Sample Collection and Preparation}

20 samples of two different fish species (Clarias gariepinus and Coptodon guineensis) were collected during harvest from fishers from Eniong River. The samples were carefully sorted out, separately contained in sterile polythene bags sealed, labeled and preserved in an ice packed boxes. The samples were immediately within (23 hours of sampling) transported to the laboratory for analysis. Representative samples of the fin-fish stocks collected were also taken to the Department of Fisheries, University of Uyo for identification.

In the Laboratory, the fin fishes were aseptically dissected. The organs (skin, gills and intestine) were removed and macerated using a sterile pestle and mortar. One gram $(1.0 \mathrm{~g})$ of each organ sample was serially diluted and used for microbiological analysis. 


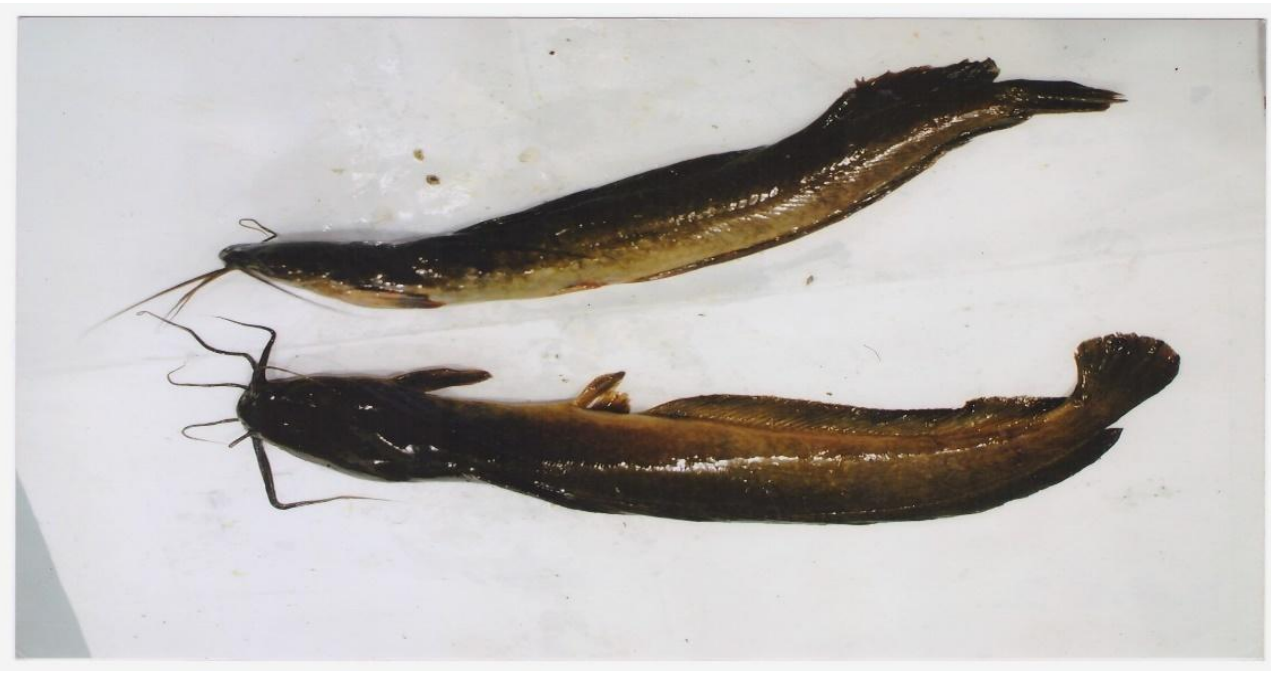

Plate I. Scientific name: Clarias gariepinus English name: Catfish Local name: Obuot

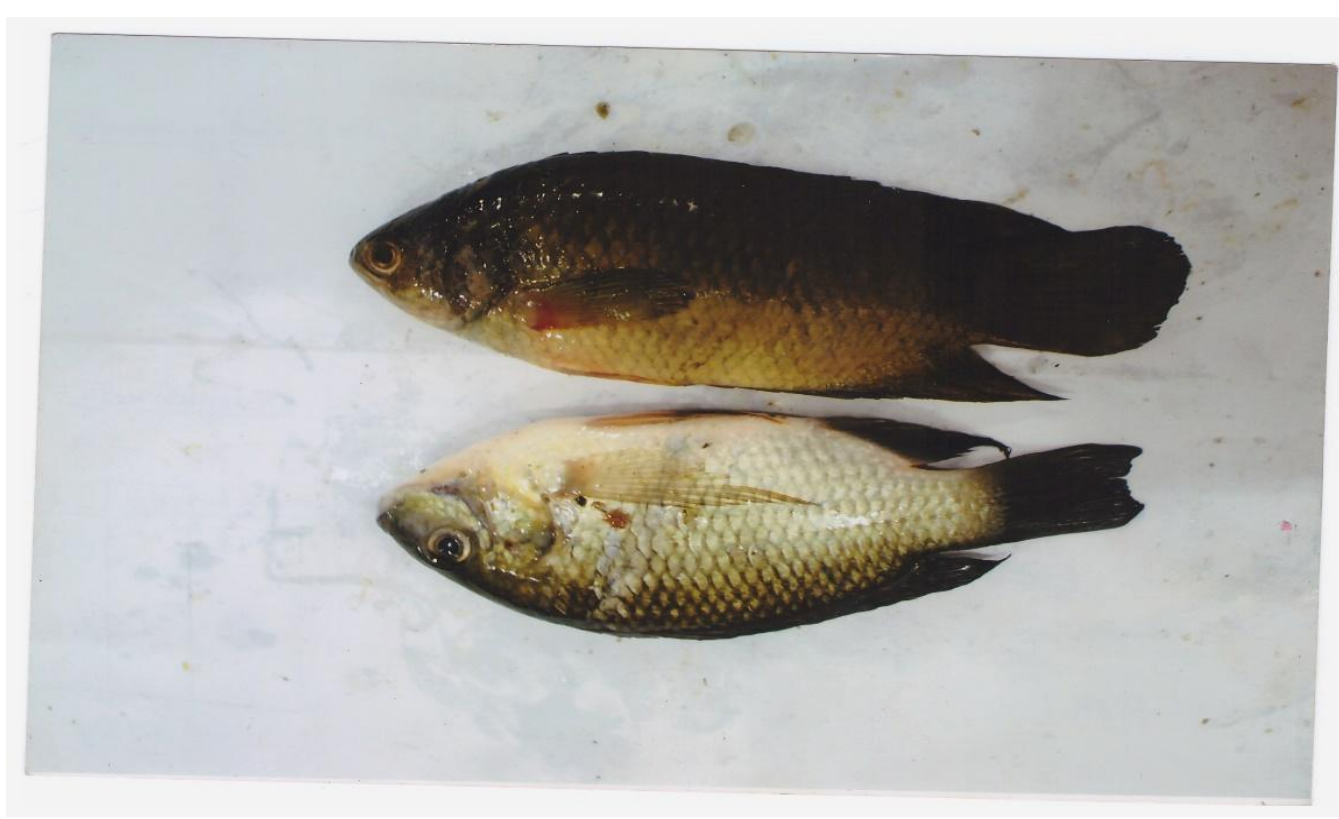

Plate II. Scientific name: Coptodon guineensis

English name: Red belly tilapia

Local name: Asat

\subsection{Analysis of Bacterial Contaminants}

This procedure was carried out to enhance the enumeration of the bacterial load of the samples. Tenfold serial dilution of $1.0 \mathrm{~g}$ of gills, tissue and intestine of each representative fish sample was carried out as described by Cheesbrough [19]. Here, $1.0 \mathrm{~g}$ of each sample was added to $9 \mathrm{ml}$ sterile water then sequentially diluted to obtain the required dilution.

The media used for the study were: Nutrient Agar (NA), MacConkey Agar (MCA), Eosine Methylene Blue Agar (EMBA) and SalmonellaShigella agar (SSA) for the enumeration and isolation of heterotrophic bacteria, total coliform, 
feacal coliform (Escherichia coli) and Salmonella and Shigella species respectively. They were aseptically prepared according to the manufacturer's instructions, sterilized by autoclaving at $121^{\circ} \mathrm{C}$ for 15 minutes.

The density of heterotrophic and potential pathogens was determined using standard analytical procedures [20]. Staphylococcus aureus, Escherichia coli (fecal coliform) and Salmonella and Shigella loads on the samples was determined using the pour plate technique [20]. All inoculated plates were incubated at $37^{\circ} \mathrm{C}$ for 24 hours.

After 24 hours, discrete colonies that appeared on the culture plates were enumerated with the aid of a Quebec colony counter and recorded as Colony Forming Units (CFU) per gram of fish sample.

The colonies obtained from the samples were characterized using standard procedure as described by Cowan and Steel [21]. The colonies were subjected to Gram's stain and various biochemical tests such as motility test, catalase test, urease test, coagulase test, citrate test, hydrogen sulphide test, sugars utilization test and MR-VP test.

\subsection{Determination of the Virulence Factor of the Bacterial Isolates}

Virulence factor of the bacterial isolates was determined by carrying out lipase, hemolysis, gelatin hydrolysis test and Dnase using the method of Aneja [22].

\subsection{Data Analysis}

The data was analyzed using simple percentage to analyze the occurrence of bacterial contaminant in the different fish sample.

\section{RESULTS AND DISCUSSION}

\subsection{Results}

\subsubsection{Microbiological properties of fin-fish samples}

The results presented in Tables $1-2$ showed that the ability of the fin-fishes to accumulate bacterial contaminants varied between the genera of fish analyzed as well as in the fish organs as the fish intestine generally accumulated more bacterial contaminants.

\section{(a) Bacterial Loads of Fin-fish Skin Samples}

Clarias gariepinus (Table 1) had the highest level of skin contamination with ranges of $2.0 \pm 0.2 \times 10^{5}$ $-4.0 \pm 0.35 \times 10^{5} \mathrm{CFU} / \mathrm{g}$ of skin scrapings. Table 2 shows bacterial load of Coptodon guineensis skin samples. The values recorded ranged between $2.2 \pm 0.2 \times 10^{5}$ and $4.0 \pm 0.35 \times 10^{5} \mathrm{CFU} / \mathrm{g}$ for heterotrophic bacteria, $1.8 \pm 0.72 \times 10^{3}$ and $3.3 \pm 0.26 \times 10^{3} \mathrm{CFU} / \mathrm{g}$ for coliform, $1.0 \pm 0.72 \times$ $10^{3}$ and $2.2 \pm 0.92 \times 10^{3} \mathrm{CFU} / \mathrm{g}$ for fecal coliform and between 0 and $1.6 \pm 0.31 \times 10^{2} \mathrm{CFU} / \mathrm{g}$ for Salmonella and Shigella.

\section{(b) Bacterial Loads of Fin-fish Gill Samples}

Analysis of the gills of Clarias gariepinus (Table 3) revealed that its heterotrophic bacterial load ranged between $2.2 \pm 0.92 \times 10^{5}$ and $3.2 \pm 0.36 \times$ $10^{5} \mathrm{CFU} / \mathrm{g}$. The total coliform counts ranged between $1.8 \pm 0.17 \times 10^{3}$ and $2.5 \pm 0.5 \times 10^{3}$ $\mathrm{CFU} / \mathrm{g}$ while the fecal coliform counts ranged between 0 and $2.0 \pm 0.17 \times 10^{3} \mathrm{CFU} / \mathrm{g}$. There were no Salmonella and Shigella except on sample CB 3, CB 5 and CB 9 with a mean count of $1.6 \pm 0.35 \times 10^{2}, 1.5 \pm 0.44 \times 10^{2}$ and $1.4 \pm$ $0.52 \times 10^{2} \mathrm{CFU} / \mathrm{g}$ respectively.

Table 4 shows bacterial load of Coptodon guineensis gills samples. The values revealed that the heterotrophic bacterial loads of the samples ranged between $2.0 \pm 0.2 \times 10^{5}$ and $3.8 \pm$ $0.44 \times 10^{5} \mathrm{CFU} / \mathrm{g}$, Total coliform counts ranged between $1.8 \pm 0.1 \times 10^{3}$ and $2.7 \pm 0.69 \times 10^{3}$ $\mathrm{CFU} / \mathrm{g}$, the fecal coliform counts ranged between $1.2 \pm 0.17 \times 10^{3}$ and $1.9 \pm 0.1 \times 10^{3} \mathrm{CFU} / \mathrm{g}$ while the Salmonella Shigella counts ranged between $1.0 \pm 0.11 \times 10^{2}$ and $1.6 \pm 0.53 \times 10^{2} \mathrm{CFU} / \mathrm{g}$.

\section{(c) Bacterial Loads of Fin-fish Intestinal Samples}

For Clarias gariepinus the results revealed that the heterotrophic bacterial densities ranged between $2.5 \pm 0.5 \times 10^{5}$ and $5.4 \pm 0.87 \times 10^{5}$ $\mathrm{CFU} / \mathrm{g}$. The total coliform counts ranged between $2.0 \pm 0.17 \times 10^{3}$ and $3.0 \pm 0.26 \times 10^{3} \mathrm{CFU} / \mathrm{g}$, while the fecal coliform counts ranged between $1.8 \pm 0.26 \times 10^{3}$ and $2.7 \pm 0.26 \times 10^{3} \mathrm{CFU} / \mathrm{g}$. The viable cells of Salmonella Shigella detected ranged between $1.4 \pm 0.52 \times 10^{2}$ and $1.8 \pm 0.26$ $x 10^{2} \mathrm{CFU} / \mathrm{g}$ respectively (Table 5 ). 
Table 1. Bacteriological loads of Clarias gariepinus skin samples

\begin{tabular}{|c|c|c|c|c|}
\hline Sample & $\begin{array}{l}\text { THBC } \\
\left(\times 10^{5} \mathrm{cfu} / \mathrm{g}\right)\end{array}$ & $\begin{array}{l}\text { Total Coliform } \\
\left(\times 10^{3} \mathrm{cfu} / \mathrm{g}\right)\end{array}$ & $\begin{array}{l}\text { Fecal Coliform } \\
\left.\text { (x } 10^{3} \mathrm{cfu} / \mathrm{g}\right)\end{array}$ & $\begin{array}{l}\text { Salmonella Shigella } \\
\left(\times 10^{2} \mathrm{cfu} / \mathrm{g}\right)\end{array}$ \\
\hline CB 1 & $3.0 \pm 0.26$ & $1.5 \pm 0.5$ & - & - \\
\hline CB 2 & $3.0 \pm 0.2$ & $2.2 \pm 0.2$ & $1.5 \pm 0.44$ & $1.4 \pm 0.52$ \\
\hline CB 3 & $2.8 \pm 0.2$ & $2.0 \pm 0.17$ & $1.5 \pm 0.44$ & - \\
\hline CB 4 & $2.9 \pm 0.80$ & $2.2 \pm 0.2$ & $1.3 \pm 0.26$ & - \\
\hline CB 5 & $3.5 \pm 0.62$ & $2.2 \pm 0.2$ & $1.4 \pm 0.52$ & - \\
\hline CB 6 & $3.2 \pm 0.36$ & $2.2 \pm 0.92$ & - & - \\
\hline CB 7 & $4.0 \pm 0.35$ & $3.3 \pm 0.26$ & $1.3 \pm 0.40$ & - \\
\hline CB 8 & $2.0 \pm 0.2$ & $1.8 \pm 0.72$ & $1.5 \pm 0.44$ & - \\
\hline CB 9 & $2.2 \pm 0.92$ & $1.8 \pm 0.72$ & $1.5 \pm 0.44$ & $1.3 \pm 0.40$ \\
\hline
\end{tabular}

Table 2. Bacteriological loads of Coptodon guineensis skin samples

\begin{tabular}{|c|c|c|c|c|}
\hline Sample & $\begin{array}{l}\text { THBC } \\
\left(\times 10^{5} \mathrm{cfu} / \mathrm{g}\right)\end{array}$ & $\begin{array}{l}\text { Total Coliform } \\
\left(\times 10^{3} \mathrm{cfu} / \mathrm{g}\right)\end{array}$ & $\begin{array}{l}\text { Fecal Coliform } \\
\left(\times 10^{3} \mathrm{cfu} / \mathrm{g}\right)\end{array}$ & $\begin{array}{l}\text { Salmonella Shigella } \\
\left(\times 10^{2} \mathrm{cfu} / \mathrm{g}\right)\end{array}$ \\
\hline CG 1 & $2.5 \pm 0.5$ & $1.8 \pm 0.72$ & $1.2 \pm 0.35$ & $1.0 \pm 0.1$ \\
\hline CG 2 & $3.3 \pm 0.53$ & $1.8 \pm 0.72$ & $1.5 \pm 0.44$ & - \\
\hline CG 3 & $4.0 \pm 0.35$ & $3.3 \pm 0.26$ & $2.0 \pm 0.26$ & - \\
\hline CG 4 & $2.6 \pm 0.66$ & $2.0 \pm 0.31$ & $1.6 \pm 0.26$ & $1.3 \pm 0.17$ \\
\hline CG 5 & $3.2 \pm 0.92$ & $1.8 \pm 0.45$ & $1.0 \pm 0.72$ & $1.0 \pm 0.26$ \\
\hline CG 6 & $2.2 \pm 0.2$ & $2.0 \pm 0.72$ & $1.4 \pm 0.69$ & $1.5 \pm 0.45$ \\
\hline CG 7 & $2.8 \pm 0.2$ & $2.1 \pm 0.45$ & $2.0 \pm 0.17$ & - \\
\hline CG 8 & $2.5 \pm 0.87$ & $2.3 \pm 0.16$ & $2.2 \pm 0.92$ & $1.6 \pm 0.31$ \\
\hline CG 9 & $3.0 \pm 0.36$ & $2.6 \pm 0.17$ & $2.2 \pm 0.35$ & $1.5 \pm 0.17$ \\
\hline
\end{tabular}

Table 3. Total bacterial count of Clarias gariepinus gill samples

\begin{tabular}{|c|c|c|c|c|}
\hline Sample & $\begin{array}{l}\text { THBC } \\
\left(\times 10^{5} \text { cfu/g) }\right.\end{array}$ & $\begin{array}{l}\text { Total Coliform } \\
\left(\times 10^{3} \mathrm{cfu} / \mathrm{g}\right)\end{array}$ & $\begin{array}{l}\text { Fecal Coliform } \\
\left(\times 10^{3} \mathrm{cfu} / \mathrm{g}\right)\end{array}$ & $\begin{array}{l}\text { Salmonella Shigella } \\
\left(\times 10^{2} \mathrm{cfu} / \mathrm{g}\right)\end{array}$ \\
\hline CB 1 & $2.6 \pm 1.58$ & $2.5 \pm 0.5$ & $1.5 \pm 0.2$ & - \\
\hline CB 2 & $2.4 \pm 0.26$ & $1.8 \pm 0.26$ & - & - \\
\hline CВ 3 & $2.7 \pm 0.36$ & $2.2 \pm 0.35$ & $1.7 \pm 0.17$ & $1.6 \pm 0.35$ \\
\hline CB 4 & $2.2 \pm 0.92$ & $2.0 \pm 0.17$ & $1.5 \pm 0.44$ & - \\
\hline CB 5 & $3.2 \pm 0.36$ & $2.0 \pm 0.2$ & $1.8 \pm 0.72$ & $1.5 \pm 0.44$ \\
\hline CB 6 & $2.8 \pm 0.2$ & $1.8 \pm 0.17$ & - & - \\
\hline CB 7 & $2.8 \pm 0.2$ & $2.0 \pm 0.17$ & $1.5 \pm 0.44$ & - \\
\hline CB 8 & $2.6 \pm 1.58$ & $2.2 \pm 0.2$ & $1.3 \pm 0.40$ & - \\
\hline CB 9 & $2.8 \pm 0.2$ & $2.1 \pm 0.3$ & $2.0 \pm 0.17$ & $1.4 \pm 0.52$ \\
\hline
\end{tabular}

Table 6 shows that the heterotrophic bacterial load of Coptodon guineensis intestinal samples ranged between $2.3 \pm 0.16 \times 10^{5}$ and $3.6 \pm 0.1 \times$ $10^{5} \mathrm{CFU} / \mathrm{g}$ while the coliform and fecal coliform counts ranged between $2.1 \pm 0.11 \times 10^{3}$ and 2.5 $\pm 0.26 \times 10^{3} \mathrm{CFU} / \mathrm{g}$ and $1.8 \pm 0.17 \times 10^{3}$ and 2.4 $\pm 0.2 \times 10^{3} \mathrm{CFU} / \mathrm{g}$ respectively. Salmonella and Shigella were detected and the values ranged between $1.0 \pm 0.17 \times 10^{2}$ and $1.9 \pm 0.26 \times 10^{2}$ $\mathrm{CFU} / \mathrm{g}$. 
d) Diverse Species of Bacteria Isolated from Fish Samples

The cultural and biochemical characteristics of the bacterial isolates are represented in Table 7 below. The attributes show that the culture-able bacteria associated with the fin-fishes were Klebsiella sp, Bacillus sp, Enterobacter, Streptococcus sp, Micrococcus sp, Lactobacillus, Serratia sp, Proteus sp, Salmonella sp, Shigella $\mathrm{sp}$, and Escherichia coli.

Table 4. Bacteriological loads of Coptodon guineensis gill samples

\begin{tabular}{|c|c|c|c|c|}
\hline Sample & $\begin{array}{l}\text { THBC } \\
\left(\times 10^{5} \text { cfu/g) }\right.\end{array}$ & $\begin{array}{l}\text { Total Coliform } \\
\left(\times 10^{3} \mathrm{cfu} / \mathrm{g}\right)\end{array}$ & $\begin{array}{l}\text { Fecal Coliform } \\
\left(\times 10^{3} \mathrm{cfu} / \mathrm{g}\right)\end{array}$ & $\begin{array}{l}\text { Salmonella Shigella } \\
\left(\times 10^{2} \mathrm{cfu} / \mathrm{g}\right)\end{array}$ \\
\hline CG 1 & $3.0 \pm 1.5$ & $2.0 \pm 0.51$ & $1.4 \pm 0.34$ & $1.0 \pm 0.12$ \\
\hline CG 2 & $2.8 \pm 0.7$ & $2.7 \pm 0.69$ & $1.8 \pm 0.17$ & $1.6 \pm 0.53$ \\
\hline CG 3 & $2.0 \pm 0.92$ & $2.0 \pm 0.44$ & $1.2 \pm 0.17$ & $1.0 \pm 0.11$ \\
\hline CG 4 & $2.4 \pm 0.26$ & $1.8 \pm 0.22$ & $1.5 \pm 0.26$ & $1.2 \pm 0.2$ \\
\hline CG 5 & $2.0 \pm 0.2$ & $1.8 \pm 0.1$ & $1.7 \pm 0.17$ & $1.3 \pm 0.53$ \\
\hline CG 6 & $2.8 \pm 0.2$ & $1.8 \pm 0.44$ & $1.5 \pm 0.72$ & $1.0 \pm 0.26$ \\
\hline CG 7 & $3.1 \pm 0.26$ & $2.5 \pm 0.26$ & $1.9 \pm 0.1$ & $1.5 \pm 0.22$ \\
\hline CG 8 & $2.4 \pm 0.27$ & $2.0 \pm 0.24$ & $1.8 \pm 0.26$ & $1.5 \pm 0.2$ \\
\hline CG 9 & $3.8 \pm 0.44$ & $2.5 \pm 0.26$ & $1.8 \pm 0.79$ & $1.2+0.45$ \\
\hline
\end{tabular}

Table 5. Bacteriological loads of Clarias gariepinus intestine samples

\begin{tabular}{|c|c|c|c|c|}
\hline Sample & $\begin{array}{l}\text { THBC } \\
\left(\times 10^{5} \mathrm{cfu} / \mathrm{g}\right)\end{array}$ & $\begin{array}{l}\text { Total Coliform } \\
\left(\times 10^{3} \mathrm{cfu} / \mathrm{g}\right)\end{array}$ & $\begin{array}{l}\text { Fecal Coliform } \\
\left(\times 10^{3} \mathrm{cfu} / \mathrm{g}\right)\end{array}$ & $\begin{array}{l}\text { Salmonella Shigella } \\
\left(\times 10^{2} \mathrm{cfu} / \mathrm{g}\right)\end{array}$ \\
\hline CB 1 & $5.4 \pm 0.87$ & $2.0 \pm 0.17$ & $1.8 \pm 0.26$ & $1.4 \pm 0.52$ \\
\hline CB 2 & $3.2 \pm 0.36$ & $3.0 \pm 0.26$ & $2.7 \pm 0.26$ & $1.8 \pm 0.26$ \\
\hline CB 3 & $3.2 \pm 0.36$ & $2.9 \pm 0.53$ & $2.0 \pm 0.26$ & $1.7 \pm 0.34$ \\
\hline CB 4 & $2.7 \pm 0.2$ & $2.6 \pm 0.36$ & $2.5 \pm 0.2$ & $1.7 \pm 0.17$ \\
\hline CB 5 & $3.0 \pm 0.3$ & $2.7 \pm 0.26$ & $2.5 \pm 0.53$ & $1.8 \pm 0.26$ \\
\hline CB 6 & $2.5 \pm 0.5$ & $2.3 \pm 0.1$ & $2.0 \pm 1$ & $1.5+0.44$ \\
\hline CB 7 & $3.2 \pm 0.36$ & $3.0 \pm 0.26$ & $2.7 \pm 0.26$ & $1.8 \pm 0.26$ \\
\hline CB 8 & $2.7 \pm 0.2$ & $2.6 \pm 0.36$ & $2.5 \pm 0.2$ & $1.7 \pm 0.17$ \\
\hline CB 9 & $3.0 \pm 0.26$ & $2.2 \pm 0.3$ & $2.1 \pm 0.26$ & $1.4 \pm 0.53$ \\
\hline
\end{tabular}

Table 6. Bacteriological loads of Coptodon guineensis intestine samples

\begin{tabular}{|c|c|c|c|c|}
\hline Sample & THBC $\left(\times 10^{5} \mathrm{cfu} / \mathrm{g}\right)$ & $\begin{array}{l}\text { Total Coliform } \\
\left(\times 10^{3} \mathrm{cfu} / \mathrm{g}\right)\end{array}$ & $\begin{array}{l}\text { Fecal Coliform } \\
\left(\times 10^{3} \mathrm{cfu} / \mathrm{g}\right)\end{array}$ & $\begin{array}{l}\text { Salmonella Shigella } \\
\left(\times 10^{2} \mathrm{cfu} / \mathrm{g}\right)\end{array}$ \\
\hline CG 1 & $3.2 \pm 0.35$ & $2.5 \pm 0.26$ & $2.4 \pm 0.2$ & $1.8 \pm 0.2$ \\
\hline CG 2 & $2.3 \pm 0.21$ & $2.1 \pm 0.17$ & $1.9 \pm 0.17$ & $1.8 \pm 0.23$ \\
\hline CG 3 & $3.0 \pm 0.1$ & $2.1 \pm 0.1$ & $2.0 \pm 0.3$ & $1.9 \pm 0.1$ \\
\hline CG 4 & $2.3 \pm 0.26$ & $2.1 \pm 0.26$ & $1.8 \pm 0.17$ & $1.4 \pm 0.27$ \\
\hline CG 5 & $3.6 \pm 0.1$ & $2.4 \pm 0.16$ & $2.1 \pm 0.3$ & $1.5 \pm 0.2$ \\
\hline CG 6 & $3.2 \pm 0.44$ & $2.2 \pm 0.2$ & $2.0 \pm 0.36$ & $1.9 \pm 0.26$ \\
\hline CG 7 & $3.0 \pm 0.1$ & $2.3 \pm 0.17$ & $1.9 \pm 0.3$ & $1.4 \pm 0.1$ \\
\hline CG 8 & $2.3 \pm 0.16$ & $2.1 \pm 0.26$ & $1.8 \pm 0.3$ & $1.0 \pm 0.17$ \\
\hline CG 9 & $3.4 \pm 0.14$ & $2.1 \pm 0.13$ & $2.0 \pm 0.17$ & $1.8 \pm 0.21$ \\
\hline
\end{tabular}

Values are mean of triplicate determination $\pm S D$

$S D=$ Standard Deviation 
Table 7. Morphological, cultural and biochemical characteristics of bacteria isolated from the fin-fish samples

\begin{tabular}{|c|c|c|c|c|c|c|c|c|c|c|c|c|c|c|c|c|}
\hline $\begin{array}{l}\text { 흥 } \\
\frac{0}{0} \\
\frac{}{2} \\
\frac{0}{2}\end{array}$ & $\begin{array}{l}\frac{5}{N} \\
\frac{5}{0} \\
\frac{5}{\pi} \\
\frac{0}{0}\end{array}$ & 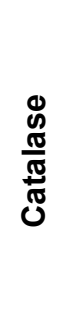 & 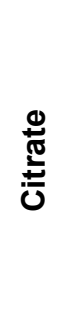 & 疍 & 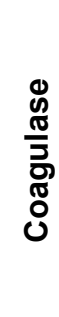 & 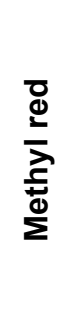 & 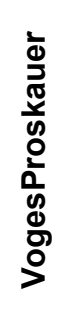 & 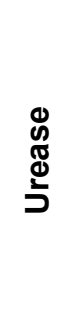 & 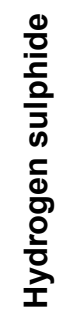 & 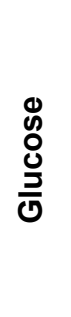 & 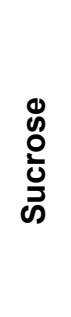 & 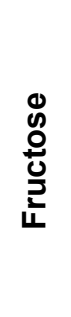 & 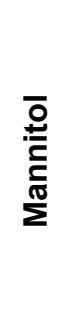 & 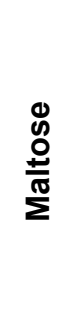 & 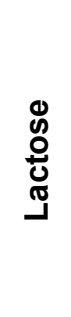 & $\begin{array}{l}\frac{E}{0} \\
\frac{0}{E} \\
\frac{\pi}{0} \\
\frac{1}{0} \\
\frac{0}{0} \\
\frac{0}{\pi} \\
\frac{0}{0} \\
\frac{0}{0}\end{array}$ \\
\hline cocci & + & + & - & - & + & - & - & + & - & AG & $A$ & $A$ & $A G$ & $A G$ & $A$ & $B$ \\
\hline Rod & & + & $\overline{+}$ & $\overline{+}$ & & $\bar{t}$ & - & + & $\bar{t}$ & $A G$ & $A G$ & & & $A G$ & $A G$ & $C$ \\
\hline Rod & $\overline{+}$ & + & + & + & - & + & - & - & - & $A$ & A & $\bar{A}$ & - & $A$ & & $\mathrm{D}$ \\
\hline Rod & - & + & + & _ & - & - & $\overline{+}$ & $\bar{t}$ & $\overline{+}$ & $A G$ & $A G$ & $A G$ & - & $A G$ & $\bar{A} G$ & $E$ \\
\hline cocci & $\overline{+}$ & - & + & - & - & $\overline{+}$ & - & + & + & - & & $A$ & - & $A$ & $A$ & $\bar{F}$ \\
\hline cocci & + & - & + & - & - & + & - & + & + & & $\bar{A}$ & A & - & A & - & $G$ \\
\hline Rod & + & - & + & $\bar{t}$ & - & + & - & - & + & $\bar{A} G$ & A & - & - & & - & $\mathrm{H}$ \\
\hline Rod & _ & - & + & + & - & _ & $\overline{+}$ & - & + & & $A G$ & $\overline{\mathrm{A}}$ & - & $\bar{A} G$ & $\bar{A} G$ & 1 \\
\hline Rod & - & - & + & + & - & $\bar{t}$ & - & $\bar{t}$ & + & $\bar{A} G$ & $A G$ & $A G$ & - & $A G$ & $A G$ & $\mathrm{~J}$ \\
\hline Rod & - & $\overline{+}$ & - & - & - & + & - & - & + & $A$ & $A$ & A & $\bar{A} G$ & $A G$ & & $\mathrm{~K}$ \\
\hline Rod & - & + & - & $\bar{t}$ & - & + & - & - & & $A G$ & A & & $A G$ & $A G$ & $\bar{A} G$ & $\mathrm{~L}$ \\
\hline Rod & - & + & $\overline{+}$ & + & & + & & - & & A & A & A & A & & & $\mathrm{M}$ \\
\hline
\end{tabular}

Key: $A=$ Acid production, $A G=$ Acid and Gas production, $B=$ Staphylococcus $s p, \bar{C}=$ Klebsiella $s p, D=$ Bacillus $s p, E=E$ Enterobacter, $F=$ Streptococcus $s p, \bar{G}=$ Micrococcus $s p, H=$ Lactobacillus sp, $I=$ Serratia $s p, J=$ Proteus $s p, K=$ Shigella $s p, L=$ Escherichia coli, $M=$ Salmonella $s p$ 
Table 8. Occurrence of bacteria on Clarias gariepinus samples

\begin{tabular}{llllll}
\hline Isolate & $\begin{array}{l}\text { Gills } \\
(\mathbf{n}=\mathbf{9})\end{array}$ & $\begin{array}{l}\text { Skin } \\
(\mathbf{n}=\mathbf{9})\end{array}$ & $\begin{array}{l}\text { Intestine } \\
(\mathbf{n}=9)\end{array}$ & $\begin{array}{l}\text { Frequency of } \\
\text { occurrence }\end{array}$ & \% of occurrence \\
\hline Staphylococcus sp & $+(7)$ & $+(5)$ & $+(5)$ & 17 & 63.0 \\
Bacillus subtilis & $+(3)$ & $+(2)$ & $+(4)$ & 9 & 33.3 \\
Bacillus cereus & $+(2)$ & $+(5)$ & $+(2)$ & 9 & 33.3 \\
Micrococcus sp & - & - & $+(2)$ & 2 & 7.41 \\
Streptococcus sp & $+(7)$ & $+(2)$ & - & 9 & 33.3 \\
Proteus sp & - & $+(4)$ & - & 4 & 14.81 \\
Serratia sp & $+(4)$ & - & - & 4 & 14.81 \\
Salmonella sp & $+(1)$ & $+(1)$ & $+(2)$ & 4 & 14.81 \\
Shigella sp & - & - & $+(3)$ & 3 & 11.1 \\
Escherichia coli & - & $+(2)$ & $+(6)$ & 8 & 29.6 \\
Enterobacter sp & $+(5)$ & $+(1)$ & $+(3)$ & 9 & 33.3 \\
Klebsiella sp & - & $+(2)$ & $+(5)$ & 7 & 26.0 \\
Lactobacillus sp & $+(2)$ & - & $+(3)$ & 5 & 18.5 \\
\hline
\end{tabular}

Table 9. Occurrence of bacteria on Coptodon guineensis samples

\begin{tabular}{llllll}
\hline Isolate & $\begin{array}{l}\text { Gills } \\
(\mathbf{n = 9})\end{array}$ & $\begin{array}{l}\text { Skin } \\
(\mathbf{n = 9})\end{array}$ & $\begin{array}{l}\text { Intestine } \\
(\mathbf{n = 9})\end{array}$ & $\begin{array}{l}\text { Frequency of } \\
\text { occurrence }\end{array}$ & $\begin{array}{l}\text { \% of } \\
\text { occurrence }\end{array}$ \\
\hline Staphylococcus sp & - & $+(9)$ & $+(3)$ & 12 & 44.4 \\
Bacillus subtilis & $+(5)$ & $+(2)$ & $+(2)$ & 9 & 33.3 \\
Bacillus cereus & $+(2)$ & $+(5)$ & $+(2)$ & 9 & 33.3 \\
Micrococcus sp & - & $+(4)$ & $+(4)$ & 8 & 29.6 \\
Streptococcus sp & $+(6)$ & $+(2)$ & - & 8 & 29.6 \\
Proteus sp & $+(3)$ & $+(6)$ & - & 9 & 33.3 \\
Serratia sp & $+(3)$ & - & - & 3 & 11.1 \\
Salmonella sp & - & - & $+(4)$ & 4 & 14.8 \\
Shigella sp & - & - & $+(3)$ & 3 & 11.1 \\
Escherichia coli & - & $+(5)$ & $+(5)$ & 10 & 37.0 \\
Enterobacter sp & $+(5)$ & $+(1)$ & $+(4)$ & 10 & 37.0 \\
Klebsiella sp & - & $+(3)$ & $+(5)$ & 8 & 29.6 \\
Lactobacillus sp & $+(3)$ & - & $+(3)$ & 6 & 22.2 \\
\hline
\end{tabular}

Table 10. Virulence attributes of the isolated bacterial species

\begin{tabular}{llllll}
\hline Isolate & Lipase & Haemolysis & DNase & Gelatinase & Coagulase \\
\hline Staphylococcus sp & - & Beta & + & + & + \\
Bacillus subtilis & - & Beta & - & + & - \\
Bacillus cereus & + & Beta & - & + & - \\
Micrococcus sp & + & Gamma & - & - & + \\
Streptococcus sp & + & Gamma & - & - & - \\
Proteus sp & + & Alpha & + & - & - \\
Salmonella sp & + & Beta & + & - & - \\
Serratia sp & + & Gamma & + & + & - \\
Shigella sp & + & Alpha & + & + & - \\
Escherichia coli & + & Beta & - & - & - \\
Enterobacter sp & + & Alpha & + & - & - \\
Klebsiellasp & - & Gamma & + & - & - \\
Lactobacillus sp & + & Alpha & - & + & - \\
\hline
\end{tabular}




\section{e) Occurrence and Prevalence of Bacterial Species on Fin-fish Samples}

Analysis of the occurrence of various bacterial isolates on the fin-fish samples of Clarias gariepinus and Coptodon guineensis are shown on Tables 8-9 respectively.

\section{f) Virulence Attributes of the Isolated Bacterial Species}

The virulence attributes of the isolates were assessed using lipase, hemolysis, DNase, gelatin and coagulase tests. The results (Table 10) of the analysis revealed that all the isolates but Staphylococcus and Klebsiella species were able to produce lipase.

\subsection{Discussion}

Fishes display an array of biotic responses such as changes in growth, distribution, abundance related to water pollution as well as has a greater potential of bioaccumulating environmental pollutants. Fin-fishes which are among the major class of fish encountered in the freshwater ecosystem of Eniong that constitute an important source of income and aquatic produce for the settlers as well as the nearby community. However, this is not without limitation in microbiological quality.

The present studies have shown that Clarias gariepinus andCoptodon guineensis harvested from Eniong River are laden with bacterial contaminants including potential pathogens. High numbers of coliforms, fecal coliform as well as the Salmonella and Shigella were found on the harvested fish samples. The level of bacterial contaminants accumulation however varied with the type of fish, and more contaminants were encountered in the fish intestines than the skin and gill. Slight variation was also noticed on the ability of the fishes to accumulate the different groups of bacterial contaminants with the skin of C. guineensis accumulating more coliforms and fecal coliforms respectively. More coliforms and fecal coliforms were found in the gills of $C$. guineensis. On the other hand, the intestine of $C$. gariepinus harbored the highest number of coliform and fecal colifom.

The high bacterial contaminants recorded for the fin-fishes was expected and is in agreement with previous report by Ajayi [23], who in his study reported a high bacterial population in catfish from fish pond in Akungba-Akoko community,
Nigeria. This he attributed to waste materials discharged into water bodies upon which the fishes inhabit/feed. The variations in the bacterial populations reported in this study is indicative of high bacteria accumulation potential of the finfishes [24] and may be attributed to various factors such as body size, feeding pattern, physiology and sediment bio-turbation characteristics of the fish samples $[25,26]$. The total heterotrophic bacterial counts of some of the fish samples exceeded the $1.2 \times 10^{5} \mathrm{cfu} / \mathrm{g}$ limit recommended by the International Commission on Microbiological Specifications for Food and World Health Organization [27] for fresh fishes. The unsafe status of the fish samples is further confirmed by the remarkable incidence of fecal coliform which indicates that the fishes are readily exposed to fresh human fecal matter.

The culture-able bacteria species associated with the fin-fish samples include Staphylococcus sp, Klebsiella sp, Bacillus sp, Enterobacter, Streptococcus sp, Micrococcus sp, Lactobacillus, Serratia sp, Proteus sp, Salmonella sp, Shigella $\mathrm{sp}$, and Escherichia coli. Similarly, many researchers [28] have reported different bacterial species from the skin of sea-water fish. Sugita et al. [29] reported that Staphylococcus sp and Escherichia coli were isolated frequently from the skin of freshwater fish. He concluded that the skin of freshwater fishes was the natural habitat of these bacteria. Some investigations reported that the skin of the Clarias species contained Klebsiella sp, Pseudomonas sp. and Micrococcus $\mathrm{sp}$ as the predominant genera. The percentage of occurrence of the isolates in the various fish samples was also found to vary with the fish species. Staphylococcus sp had the highest rate of occurrence of $63 \%$ and $44.4 \%$ in C. gariepinus and C. guineensis respectively while the least prevalent organism was Micrococussp with $7.41 \%$, and Serratia and Shigella sp with $11.1 \%$ for $C$. gariepinus and $C$. guineensis respectively.

Virulence is the degree to which a microbe can cause damage to its host. In this study, it was pertinent to assess the degree of virulence of each of the bacterial species isolated from the fish samples. This was achieved by assaying the isolates for the production of various enzymes such as lipase, hemolysin, DNase, gelatinase and coagulase. The analysis revealed that each of the isolates exhibited varying degree of virulence. Of the 13 bacterial isolates obtained from the various fish samples, ten (Bacillus cereus, Micrococcus sp, Streptococcus sp, 
Proteus sp, Serratia sp, Salmonella sp, Shigella $\mathrm{sp}$, E. coli, Enterobacter $\mathrm{sp}$ and Lactobacillus $\mathrm{sp}$ had the potentials to produce lipases enzyme. This enzyme is responsible for breaking down lipids.

Hemolytic test is used to screen for the ability of the isolates to produce hemolysis, an enzyme that breaks down hemoglobin. Hemolytic ability of isolates is usually categorized into three partial (alpha hemolysis), complete (beta hemolysis) and no hemolysis (gamma hemolysis). The result of the hemolytic test of the isolates obtained from the fish samples revealed that, four (Proteus sp, Shigella sp, Enterobacter $\mathrm{sp}$ and Lactobacillus sp) where able to partially lyse hemoglobin completely (alpha hemolysis), five (Staphylococcus sp, B. subtilis, B. cereus, Salmonella $\mathrm{sp}$ and E.coli) were able to completely lyse hemoglobin (beta hemolysis) whereas Micrococcus sp., Streptococcus sp., Serratia sp and Klebsiella sp were unable to lyse hemoglobin at all (gamma hemolysis). On the other hand seven of the isolates were able to produce DNase, an enzyme that degrades DNA molecules. Only two (Staphylococcus sp and Micrococcus sp) of the isolates were able to produce coagulase. Based on this result, the isolate with the highest degree of virulence was Staphylococcus sp.

\section{CONCLUSION}

The result of this study have revealed that the fin fishes harbour a high population of diverse bacteria including pathogenic strains of Klebsiella $\mathrm{sp}$, Bacillus sp, Enterobacter, Serratia sp, Proteus sp, Salmonella sp, Staphylococcus aureus, Shigella sp, and Escherichia coli which are commonly associated with human and infant gastroenteritis. Bacterial contaminant accumulation varied with the fish types and also with the parts of fish analyzed as the intestine harboured more contaminants than the skin or gill.

The results of this study call for proper processing of aquatic foods obtained from the apparently contaminated water body.

\section{COMPETING INTERESTS}

Authors have declared that no competing interests exist.

\section{REFERENCES}

1. Lévêque $\mathrm{C}$, Balian $\mathrm{EV}$, Martens $\mathrm{K}$. An assessment of animal species diversity in continental waters. In: Segers, $H$. and Martens, K. (eds.). The diversity of aquatic ecosystems. Hydrobiologia. 2005;542:3967.

2. Dudgeon D, Arthington $\mathrm{AH}$, Gessner $\mathrm{MO}$, Kawabata ZI, Knowler DJ, Lévêque C, Naiman RJ, Prieur-Richard AH, Soto D, Stiassny MLJ, Sullivan CA. Freshwater biodiversity: Importance, threats, status and conservation challenges. Biological Review. 2006;8:1163-182.

3. Millennium Ecosystem Assessment. Ecosystems and human well-being: Wetlands and water synthesis. World Resources Institute, Washington, DC; 2005.

4. Lipp EK, Rose JB. The role of seafood in food-borne diseases in the United States of America. Review of Science and Technology. 1997;16:620-640.

5. Cahill MM. Bacterial flora of fishes: A review. Journal Microbiology Ecology. 1990;19(1):21-41.

6. Claucas IJ, Ward AR. Post-harvest fisheries development: A guide to handling, preservation, processing and quality. Charthan, Maritime, Kent ME4 4TB. United Kingdom; 1996.

7. Kvenberg EJ. Non-indigenous bacterial pathogen. In: Donn R, Cameron $\mathrm{H}$, Van Nostrand R. (Eds). Microbiology of marine food products. New York. 1991;263-291.

8. Rodricks EG. Indigenous pathogen: Vibrionaceae. Microbiology of Marine Food Products, New York, Reinhold. 1991;285295.

9. Austin B. Taxonomy of bacterial fish pathogens. Veterinary Research. 2011; 42(1):20.

10. Green BW, Teichert-Coddington DR. Production of Oreochromis niloticus fry for hormonal sex reversal in relation to water temperature. Journal of Applied Ichthyology. 1993;9:230-236.

11. Musaiger AO, Souza R. Chemical composition of raw fish consumed in Bahrain. Pak Journal of Biological Science. 2008;11:55-61.

12. Christopher AE, Vincent O, Grace I, Rebecca E, Joseph E. Distribution of heavy metals in bones, gills, livers and muscles of (Tilapia) Oreochromis niloticus from Henshaw Town Beach Market in Calabar Nigeria. Pak. Journal Nutrition. 2009;8:1209-1211.

13. FAO. Recycling of animal wastes as a source of nutrient for freshwater fish 
culture within an integrated livestock system; 2003.

Available:http://www.fao.org/docrep/field/0 03/A C526E/AC526E01.htm

14. Abdelhamid AM, Gawish MM, Soryal KA. Comparative study between desert cultivated and natural fisheries of mullet fish in Egypt. II-Microbiological Concern. Journal of Agricultural Science. 2006;3: 5681-5687.

15. Mhango M, Mpuchane S, Gashe B. Incidence of indicator organisms, opportunistic and pathogenic bacteria in fish. African Journal of Food, Agriculture, Nutrition and Development. 2010;10(10): 4202-4218.

16. Ochman H, Lawrence JG, Groisman EA. Lateral gene transfer and the nature of bacterial innovation. Nature. 2000;405: 299-304.

17. Budiati T, Rusul G, Wan-Abdullah WN, Ahmad R, Arip YM. Microbiological quality of catfish (Clarias gariepinus) and tilapia (Tilapia Mossambica) obtained from wet markets and ponds in Malaysia. J Aquac Res Development. 2015;6:291.

DOI: 10.4172/2155-9546.1000291

18. Rahimi E, Shakerian A, Falavarjani AG. Prevalence and antimicrobial resistance of Salmonella isolated from fish, shrimp, lobster, and crab in Iran. Compara. Clini. Pathol. 2013;22(1):59-62.

19. Chessbrough, M. District laboratory practice in tropical countries. United Kingdom, Cambridge University Press. 2006;416.

20. Harrigan WF, McCance ME. Laboratory methods in food and diary microbiology. London: Academic Press. 1990;210.

21. Cowan ST, Steel J. Manual for identification for medical bacteria $\left(3^{\text {rd }} \mathrm{Ed}\right)$
Cambridge, U. K: Cambridge University Press. 2003;31.

22. Aneja KR. Experiments in microbiology, plant pathology and biotechnology. New Delhi: New Age International (P) Ltd. 2003;178.

23. Ajayi AO. Bacteriological study of Catfish, Clarias gariepinus, from fish pond sources in Akungba-Akoko community, Nigeria. British Microbiology Research Journal. 2012;2(1):1-9.

24. Hunt DA. Indicators of quality of shellfish waters. In A.W. Hoadly \& B.J. Dutka (Eds.), Bacterial Indicators/ Health Hazards Associated with Water ( $9^{\text {th }}$ ed.), USA. 1974;337-345.

25. FAO. The State of World Fisheries and Aquaculture. Food and Agriculture Organization of the United Nations. Fisheries and Aquaculture Department. Rome, Italy; 2012.

26. FFSG. (Freshwater Fish Specialist Group) Importance of Freshwater Fishes. FFSG Newsletter Hosted by Chester Zoo; 2013. Available:http://www.iucnffsg.org/freshwate r-fishes/importance-of-freshwater-fishes.

27. WHO (World Health Organization). Guidelines for Drinking Water Quality. Health and other supportive criteria $\left(2^{\text {nd }}\right.$ ed.), Geneva. 1996;11:39.

28. Okaeme AN. Fish diseases prevention and control. Paper Presented at the VCN Professional Country Education Seminar, Akure, 2006;8:1-17.

29. Sugita HN, Matsuo $Y$, Hirose $M$, Iwato $Y$, Deguchi A. Vibrio species strain NM10 with an inhibitory effect against Pasteurella piscicida from the intestine of Japanese coastal fish. Applied Environmental Microbiology. 1997;63:4986-4989.

(c) 2017 Umana et al.; This is an Open Access article distributed under the terms of the Creative Commons Attribution License (http://creativecommons.org/licenses/by/4.0), which permits unrestricted use, distribution, and reproduction in any medium, provided the original work is properly cited.

Peer-review history:

The peer review history for this paper can be accessed here: http://sciencedomain.org/review-history/21373 